\title{
A Spectral Element Reduced Basis Method for Navier-Stokes Equations with Geometric Variations
}

\author{
Martin W. Hess, Annalisa Quaini, and Gianluigi Rozza
}

\section{Introduction and Motivation}

Spectral element methods (SEM) use high-order polynomial ansatz functions to solve partial differential equations (PDEs) in all fields of science and engineering, see, e.g., [4-7, 12, 16] and references therein for an overview. Typically, an exponential error decay under p-refinement is observed, which can provide an enhanced accuracy over standard finite element methods at the same computational cost. In the following, we assume that the discretization error is much smaller than the model reduction error, small enough not to interfere with our results. In general, this needs to be established with the use of suitable error estimation and adaptivity techniques.

We consider the flow through a channel with a narrowing of variable height. A reduced order model (ROM) is computed from a few high-order SEM solves, which accurately approximates the high-order solutions for the parameter range of interest, i.e., the different narrowing heights under consideration. Since the parametric variations are affine, a mapping to a reference domain is applied without further interpolation techniques. The focus of this work is to show how to use simulations arising from the SEM solver Nektar++ [3] in a ROM context. In particular, the multilevel static condensation of the high-order solver is not applied, but the ROM projection works with the system matrices in local coordinates. See [12] for further details. This is in contrast to our previous work [8], since numerical

M. W. Hess $(\bowtie) \cdot$ G. Rozza

SISSA mathLab, International School for Advanced Studies, Trieste, Italy

e-mail: mhess@ sissa.it; gianluigi.rozza@ sissa.it

\author{
A. Quaini \\ Department of Mathematics, University of Houston, Houston, TX, USA \\ e-mail: quaini@math.uh.edu
}


experiments have shown that the multilevel static condensation is inefficient in a ROM context. Additionally, we consider affine geometry variations. With SEM as discretization method, we use global approximation functions for the high-order as well as reduced-order methods. The ROM techniques described in this paper are implemented in open-source project ITHACA-SEM. ${ }^{1}$

The outline of the paper is as follows. In Sect. 2, the model problem is defined and the geometric variations are introduced. Section 3 provides details on the spectral element discretization, while Sect. 4 describes the model reduction approach and shows the affine mapping to the reference domain. Numerical results are given in Sect. 5, while Sect. 6 summarizes the work and points out future perspectives.

\section{Problem Formulation}

Let $\Omega \in \mathbb{R}^{2}$ be the computational domain. Incompressible, viscous fluid motion in spatial domain $\Omega$ over a time interval $(0, T)$ is governed by the incompressible Navier-Stokes equations with vector-valued velocity $\mathbf{u}$, scalar-valued pressure $p$, kinematic viscosity $v$ and a body forcing $\mathbf{f}$ :

$$
\begin{aligned}
\frac{\partial \mathbf{u}}{\partial t}+\mathbf{u} \cdot \nabla \mathbf{u} & =-\nabla p+v \Delta \mathbf{u}+\mathbf{f}, \\
\nabla \cdot \mathbf{u} & =0 .
\end{aligned}
$$

Boundary and initial conditions are prescribed as

$$
\begin{aligned}
\mathbf{u}=\mathbf{d} & \text { on } \Gamma_{D} \times(0, T), \\
\nabla \mathbf{u} \cdot \mathbf{n}=\mathbf{g} \quad \text { on } \Gamma_{N} \times(0, T), & \\
\mathbf{u}=\mathbf{u}_{0} & \text { in } \Omega \times 0,
\end{aligned}
$$

with $\mathbf{d}, \mathbf{g}$ and $\mathbf{u}_{0}$ given and $\partial \Omega=\Gamma_{D} \cup \Gamma_{N}, \Gamma_{D} \cap \Gamma_{N}=\emptyset$. The Reynolds number $R e$, which characterizes the flow [11], depends on $v$, a characteristic velocity $U$, and a characteristic length $L$ :

$$
R e=\frac{U L}{v}
$$

We are interested in computing the steady states, i.e., solutions where $\frac{\partial \mathbf{u}}{\partial t}$ vanishes. The high-order simulations are obtained through time-advancement, while the ROM solutions are obtained with a fixed-point iteration.

\footnotetext{
$\overline{{ }^{1} \text { https://github.com/mathLab/ITHACA-SEM. }}$
} 


\subsection{Oseen-Iteration}

The Oseen-iteration is a secant modulus fixed-point iteration, which in general exhibits a linear rate of convergence [2]. Given a current iterate (or initial condition) $\mathbf{u}^{k}$, the next iterate $\mathbf{u}^{k+1}$ is found by solving linear system:

$$
\begin{aligned}
-v \Delta \mathbf{u}^{k+1}+\left(\mathbf{u}^{k} \cdot \nabla\right) \mathbf{u}^{k+1}+\nabla p & =\mathbf{f} \text { in } \Omega, \\
\nabla \cdot \mathbf{u}^{k+1} & =0 \text { in } \Omega, \\
\mathbf{u}^{k+1} & =\mathbf{d} \quad \text { on } \Gamma_{D}, \\
\nabla \mathbf{u}^{k+1} \cdot \mathbf{n} & =\mathbf{g} \text { on } \Gamma_{N} .
\end{aligned}
$$

Iterations are typical stopped when the relative difference between iterates falls below a predefined tolerance in a suitable norm, like the $L^{2}(\Omega)$ or $H_{0}^{1}(\Omega)$ norm.

\subsection{Model Description}

We consider the reference computational domain shown in Fig. 1, which is decomposed into 36 triangular spectral elements. The spectral element expansion uses modal Legendre polynomials of the Koornwinder-Dubiner type of order $p=11$ for the velocity. Details on the discretization method can be found in chapter 3.2 of [12]. The pressure ansatz space is chosen of order $p-2$ to fulfill the inf-sup stability condition $[1,20]$. A parabolic inflow profile is prescribed at the inlet (i.e., $x=0)$ with horizontal velocity component $u_{x}(0, y)=y(3-y)$ for $y \in[0,3]$. At the outlet (i.e., $x=8$ ) we impose a stress-free boundary condition, everywhere else we prescribe a no-slip condition.

The height of the narrowing in the reference configuration is $\mu=1$, from $y=1$ to $y=2$. See Fig. 1. Parameter $\mu$ is considered variable in the interval $\mu \in[0.1,2.9]$. The narrowing is shrunken or expanded as to maintain the geometry

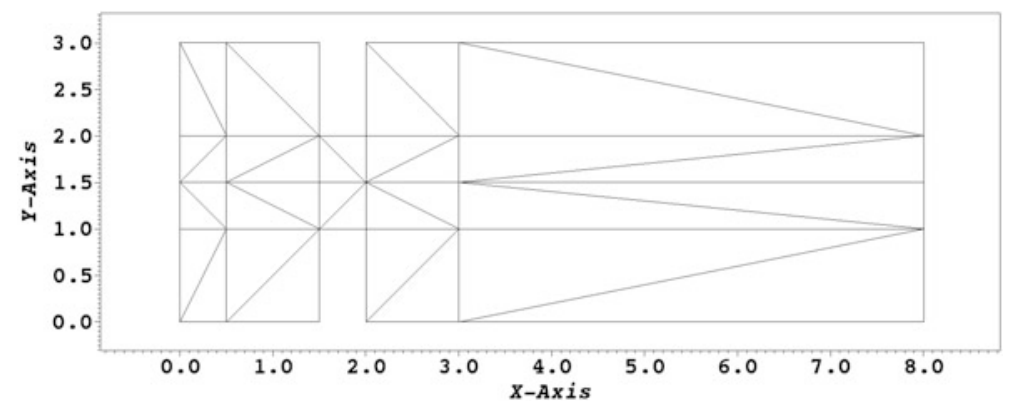

Fig. 1 Reference computational domain for the channel flow, divided into 36 triangles 

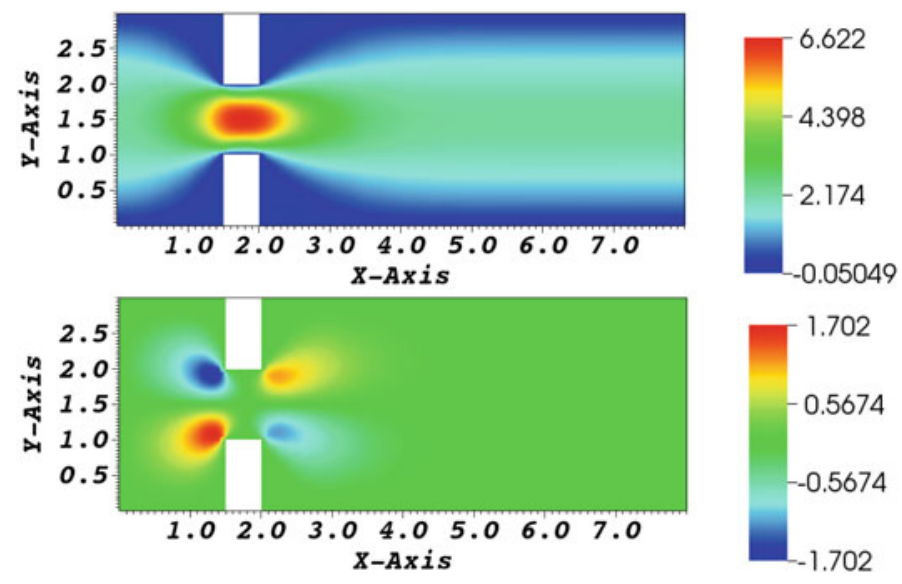

Fig. 2 Full order, steady-state solution for $\mu=1$ : velocity in x-direction (top) and y-direction (bottom)

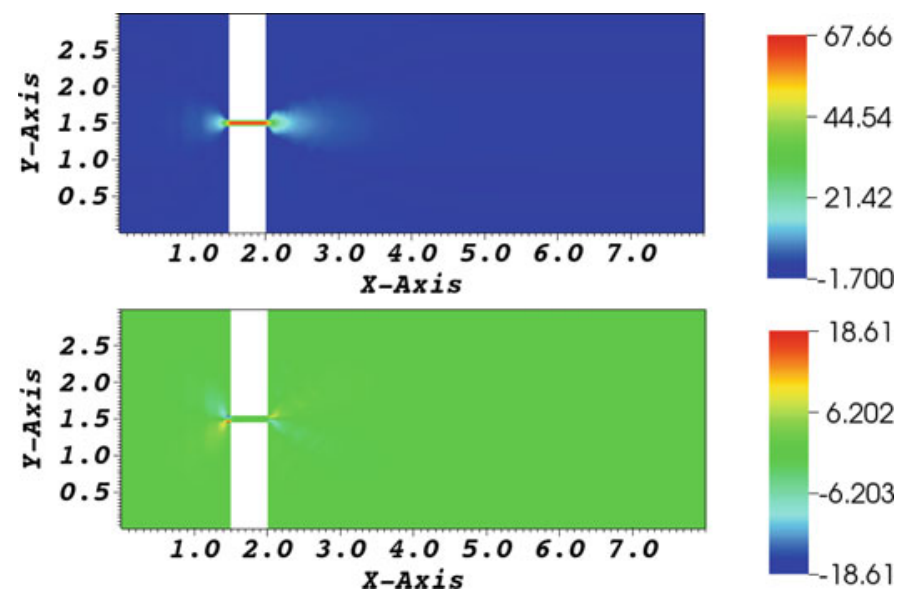

Fig. 3 Full order, steady-state solution for $\mu=0.1$ : velocity in x-direction (top) and y-direction (bottom)

symmetric about line $y=1.5$. Figures 2,3 , and 4 show the velocity components close to the steady state for $\mu=1,0.1,2.9$, respectively.

The viscosity is kept constant to $v=1$. For these simulations, the Reynolds number (6) is between 5 and 10, with maximum velocity in the narrowing as characteristic velocity $U$ and the height of the narrowing characteristic length $L$. For larger Reynolds numbers (about 30), a supercritical pitchfork bifurcation occurs giving rise to the so-called Coanda effect [8, 9, 22], which is not subject of the current study. Our model is similar to the model considered in $[17,18]$, i.e. an 


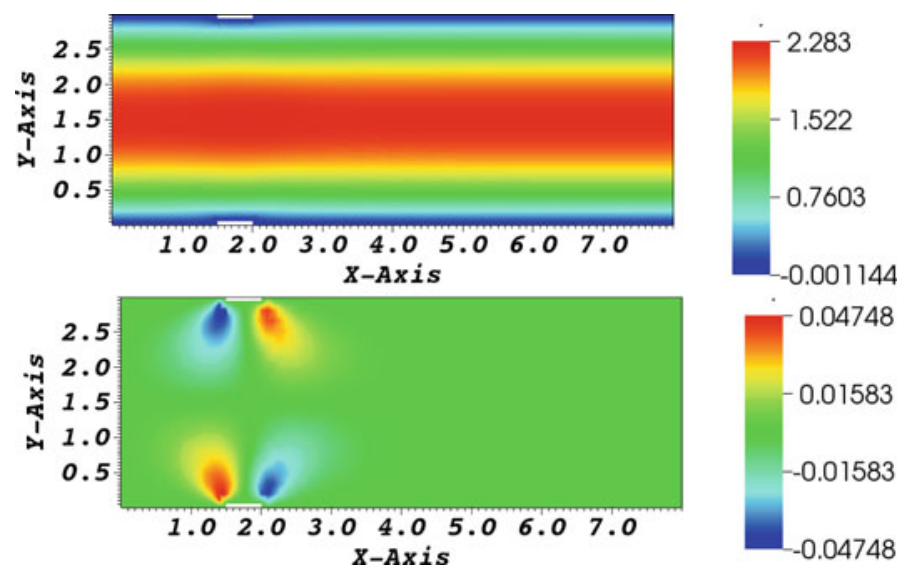

Fig. 4 Full order, steady-state solution for $\mu=2.9$ : velocity in $\mathrm{x}$-direction (top) and y-direction (bottom)

expansion channel with an inflow profile of varying height. However, in [18] the computational domain itself does not change.

\section{Spectral Element Full Order Discretization}

The Navier-Stokes problem is discretized with the spectral element method. The spectral/hp element software framework used is Nektar++ in version 4.4.0. ${ }^{2}$ The discretized system of size $N_{\delta}$ to solve at each step of the Oseen-iteration for fixed $\mu$ can be written as

$$
\left[\begin{array}{ccc}
A & -D_{b n d}^{T} & B \\
-D_{b n d} & 0 & -D_{i n t} \\
\tilde{B}^{T} & -D_{i n t}^{T} & C
\end{array}\right]\left[\begin{array}{c}
\mathbf{v}_{b n d} \\
\mathbf{p} \\
\mathbf{v}_{i n t}
\end{array}\right]=\left[\begin{array}{c}
\mathbf{f}_{b n d} \\
\mathbf{0} \\
\mathbf{f}_{i n t}
\end{array}\right],
$$

where $\mathbf{v}_{\text {bnd }}$ and $\mathbf{v}_{\text {int }}$ denote velocity degrees of freedom on the boundary and in the interior of the domain, respectively, while $\mathbf{p}$ denotes the pressure degrees of freedom. The forcing terms on the boundary and interior are denoted by $\mathbf{f}_{\text {bnd }}$ and $\mathbf{f}_{i n t}$, respectively. The matrix $A$ assembles the boundary-boundary coupling, $B$ the boundary-interior coupling, $\tilde{B}$ the interior-boundary coupling, and $C$ assembles the interior-interior coupling of elemental velocity ansatz functions. In the case of a Stokes system, it holds that $B=\tilde{B}^{T}$, but this is not the case for the Oseen equation because of the linearized convective term. The matrices $D_{b n d}$

\footnotetext{
${ }^{2}$ See www.nektar.info.
} 
and $D_{i n t}$ assemble the pressure-velocity boundary and pressure-velocity interior contributions, respectively.

The linear system (7) is assembled in local degrees of freedom, resulting in block matrices $A, B, \tilde{B}, C, D_{b n d}$ and $D_{i n t}$, each block corresponding to a spectral element. This allows for an efficient matrix assembly since each spectral element is independent from the others, but makes the system singular. In order to solve the system, the local degrees of freedom need to be gathered into the global degrees of freedom [12].

The high-order element solver Nektar++ uses a multilevel static condensation for the solution of linear systems like (7). Since static condensation introduces intermediate parameter-dependent matrix inversions (such as $C^{-1}$ in this case) several intermediate projection spaces need to be introduced to use model order reduction [8]. This can be avoided by instead projecting the expanded system (7) directly. The internal degrees of freedom do not need to be gathered, since they are the same in local and global coordinates. Only ansatz functions extending over multiple spectral elements need to be gathered.

Next, we will take the boundary-boundary coupling across element interfaces into account. Let $M$ denote the rectangular matrix which gathers the local boundary degrees of freedom into global boundary degrees of freedom. Multiplication of the first row of (7) by $M^{T} M$ will then set the boundary-boundary coupling in local degrees of freedom:

$$
\left[\begin{array}{ccc}
M^{T} M A & -M^{T} M D_{b n d}^{T} & M^{T} M B \\
-D_{b n d} & 0 & -D_{i n t} \\
\tilde{B}^{T} & -D_{i n t}^{T} & C
\end{array}\right]\left[\begin{array}{c}
\mathbf{v}_{b n d} \\
\mathbf{p} \\
\mathbf{v}_{\text {int }}
\end{array}\right]=\left[\begin{array}{c}
M^{T} M \mathbf{f}_{b n d} \\
\mathbf{0} \\
\mathbf{f}_{\text {int }}
\end{array}\right] .
$$

The action of the matrix in (8) on the degrees of freedom on the Dirichlet boundary is computed and added to the right hand side. Such degrees of freedom are then removed from (8). The resulting system can then be used in a projectionbased ROM context [13], of high-order dimension $N_{\delta} \times N_{\delta}$ and depending on the parameter $\mu$ :

$$
\mathcal{A}(\mu) \mathbf{x}(\mu)=\mathbf{f} .
$$

\section{Reduced Order Model}

The reduced order model (ROM) computes accurate approximations to the highorder solutions in the parameter range of interest, while greatly reducing the overall computational time. This is achieved by two ingredients. First, a few high-order solutions are computed and the most significant proper orthogonal decomposition (POD) modes are obtained [13]. These POD modes define the reduced order ansatz space of dimension $N$, in which the system is solved. Second, to reduce 
the computational time, an offline-online computational procedure is used. See Sect. 4.1.

The POD computes a singular value decomposition of the snapshot solutions to $99.99 \%$ of the most dominant modes [10], which define the projection matrix $U \in \mathbb{R}^{N_{\delta} \times N}$ used to project system (9):

$$
U^{T} \mathcal{A}(\mu) U \mathbf{x}_{N}(\mu)=U^{T} \mathbf{f} .
$$

The low order solution $\mathbf{x}_{N}(\mu)$ then approximates the high order solution as $\mathbf{x}(\mu) \approx U \mathbf{x}_{N}(\mu)$.

\subsection{Offline-Online Decomposition}

The offline-online decomposition [10] enables the computational speed-up of the ROM approach in many-query scenarios. It relies on an affine parameter dependency, such that all computations depending on the high-order model size can be moved into a parameter-independent offline phase, while having a fast inputoutput evaluation online.

In the example under consideration here, the parameter dependency is already affine and a mapping to the reference domain can be established without using an approximation technique such as the empirical interpolation method. Thus, there exists an affine expansion of the system matrix $\mathcal{A}(\mu)$ in the parameter $\mu$ as

$$
\mathcal{A}(\mu)=\sum_{i=1}^{Q} \Theta_{i}(\mu) \mathcal{A}_{i} .
$$

The coefficients $\Theta_{i}(\mu)$ are computed from the mapping $\mathbf{x}=T_{k}(\mu) \hat{\mathbf{x}}+\mathbf{g}_{k}$, $T_{k} \in \mathbb{R}^{2 \times 2}, \mathbf{g}_{k} \in \mathbb{R}^{2}$, which maps the deformed subdomain $\hat{\Omega}_{k}$ to the reference subdomain $\Omega_{k}$. See also $[19,21]$. Figure 5 shows the reference subdomains $\Omega_{k}$ for the problem under consideration.

For each subdomain $\hat{\Omega}_{k}$ the elemental basis function evaluations are transformed to the reference domain. For each velocity basis function $\mathbf{u}=\left(u_{1}, u_{2}\right), \mathbf{v}=$ $\left(v_{1}, v_{2}\right), \mathbf{w}=\left(w_{1}, w_{2}\right)$ and each (scalar) pressure basis function $\psi$, we can write the transformation with summation convention as:

$$
\begin{gathered}
\int_{\hat{\Omega}_{k}} \frac{\partial \hat{\mathbf{u}}}{\partial \hat{x}_{i}} \hat{v}_{i j} \frac{\partial \hat{\mathbf{v}}}{\partial \hat{x}_{j}} d \hat{\Omega}_{k}=\int_{\Omega_{k}} \frac{\partial \mathbf{u}}{\partial x_{i}} v_{i j} \frac{\partial \mathbf{v}}{\partial x_{j}} d \Omega_{k}, \\
\int_{\hat{\Omega}_{k}} \hat{\psi} \nabla \cdot \hat{\mathbf{u}} d \hat{\Omega}_{k}=\int_{\Omega_{k}} \psi \chi_{i j} \frac{\partial u_{j}}{\partial x_{i}} d \Omega_{k}, \\
\int_{\hat{\Omega}_{k}}(\hat{\mathbf{u}} \cdot \nabla) \hat{\mathbf{v}} \cdot \hat{\mathbf{w}} d \hat{\Omega}_{k}=\int_{\Omega_{k}} u_{i} \pi_{i j} \frac{\partial v_{j}}{\partial x_{i}} \mathbf{w} d \Omega_{k},
\end{gathered}
$$




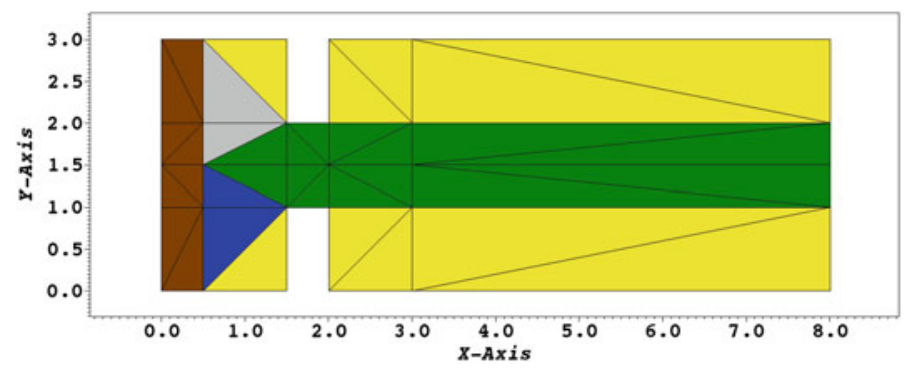

Fig. 5 Reference computational domain with subdomains $\Omega_{1}$ (green), $\Omega_{2}$ (yellow), $\Omega_{3}$ (blue), $\Omega_{4}$ (grey) and $\Omega_{5}$ (brown)

with

$$
\begin{aligned}
& v_{i j}=T_{i i^{\prime}} \hat{v}_{i^{\prime} j^{\prime}} T_{j j^{\prime}} \operatorname{det}(T)^{-1}, \\
& \chi_{i j}=\pi_{i j}=T_{i j} \operatorname{det}(T)^{-1} .
\end{aligned}
$$

The subdomain $\Omega_{5}$ (see Fig. 5) is kept constant, so that no interpolation of the inflow profile is necessary. To achieve fast reduced order solves, the offline-online decomposition expands the system matrix as in (11) and computes the parameter independent projections offline, which are stored as small-sized matrices of the order $N \times N$. Since in an Oseen-iteration each matrix is dependent on the previous iterate, the submatrices corresponding to each basis function are assembled and then formed online using the reduced basis coordinate representation of the current iterate. This is the same procedure used for the assembly of the nonlinear term in the Navier-Stokes case [13].

\section{Numerical Results}

The accuracy of the ROM is assessed using 40 snapshots sampled uniformly over the parameter domain $[0.1,2.9]$ for the POD and 40 randomly chosen parameter locations to test the accuracy. Figure 6 (left) shows the decay of the energy of the POD modes. To reach the typical threshold of $99.99 \%$ on the POD energy, it takes 9 POD modes as RB ansatz functions. Figure 6 (right) shows the relative $L^{2}(\Omega)$ approximation error of the reduced order model with respect to the full order model up to 6 digits of accuracy, evaluated at the 40 randomly chosen verification parameter locations. With 9 POD modes the maximum approximation error is less than $0.7 \%$ and the mean approximation error is less than $0.5 \%$.

While the full-order solves were computed with Nektar++, the reduced-order computations were done in ITHACA-SEM with a separate python code. To assess the computational gain, the time for a fixed point iteration step using the full- 

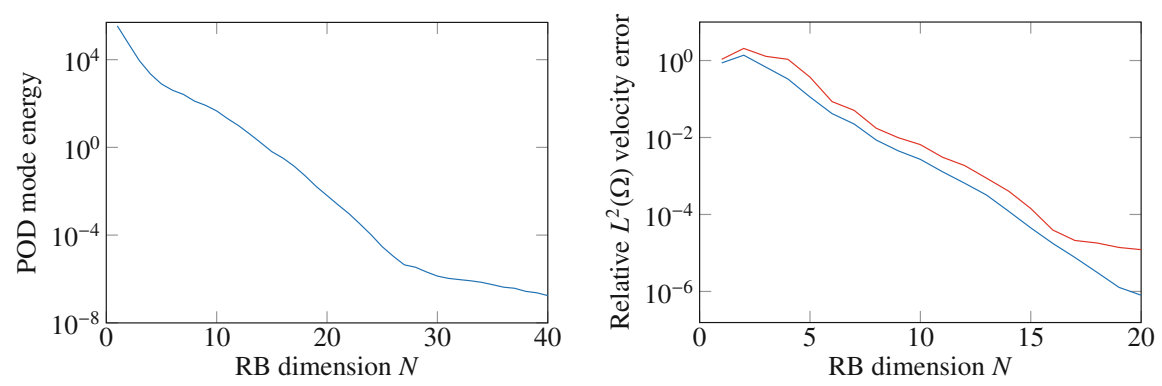

Fig. 6 Left: Decay of POD mode energy. Right: Maximum (red) and mean (blue) relative $L^{2}(\Omega)$ error for the velocity over increasing reduced basis dimension

order system is compared to the time for a fixed point iteration step of the ROM with dimension 20, both done in python. The ROM online phase reduces the computational time by a factor of over 100. The offline time is dominated by computing the snapshots and the trilinear forms used to project the advection terms. See [13] for detailed explanations.

\section{Conclusion and Outlook}

We showed that the POD reduced basis technique generates accurate reduced order models for SEM discretized models under parametric variation of the geometry. The potential of a high-order spectral element method with a reduced basis ROM is the subject of current investigations. See also [6]. Since each spectral element comprises a block in the system matrix in local coordinates, a variant of the reduced basis element method (RBEM) [14, 15] can be successfully applied in the future.

Acknowledgements This work was supported by European Union Funding for Research and Innovation through the European Research Council (project H2020 ERC CoG 2015 AROMACFD project 681447, P.I. Prof. G. Rozza). This work was also partially supported by NSF through grant DMS-1620384 (Prof. A. Quaini).

\section{References}

1. Boffi, D., Brezzi F., Fortin, M.: Mixed Finite Element Methods and Applications. Springer Series in Computational Mathematics. Springer, Berlin (2013)

2. Burger, M.: Numerical Methods for Incompressible Flow. Lecture Notes. UCLA, Los Angeles (2010) 
3. Cantwell, C.D., Moxey, D., Comerford, A., Bolis, A., Rocco, G., Mengaldo, G., de Grazia, D., Yakovlev, S., Lombard, J.-E., Ekelschot, D., Jordi, B., Xu, H., Mohamied, Y., Eskilsson, C., Nelson, B., Vos, P., Biotto, C., Kirby, R.M., Sherwin, S.J.: Nektar++: an open-source spectral/hp element framework. Comput. Phys. Commun. 192, 205-219 (2015)

4. Canuto, C., Hussaini, M.Y., Quarteroni, A., Zhang, Th.A.: Spectral Methods Fundamentals in Single Domains. Scientific Computation. Springer, Berlin (2006)

5. Canuto, C., Hussaini, M.Y., Quarteroni, A., Zhang, Th.A.: Spectral Methods Evolution to Complex Geometries and Applications to Fluid Dynamics. Scientific Computation. Springer, Berlin (2007)

6. Fick, L., Maday, Y., Patera A., Taddei T.: A stabilized POD model for turbulent flows over a range of Reynolds numbers: optimal parameter sampling and constrained projection. J. Comput. Phys. 371, 214-243 (2018)

7. Herrero, H., Maday, Y., Pla, F.: RB (Reduced Basis) for RB (Rayleigh-Bénard). Comput. Meth. Appl. Mech. Eng. 261-262, 132-141 (2013)

8. Hess, M.W., Rozza, G.: A spectral element reduced basis method in parametric CFD. In: Numerical Mathematics and Advanced Applications ENUMATH 2017. Springer, Berlin (2018, in press). E-print arXiv:1712.06432

9. Hess, M.W., Alla, A., Quaini, A., Rozza, G., Gunzburger, M.: A localized reducedorder modeling approach for PDEs with bifurcating solutions. In: Computer Methods in Applied Mechanics and Engineering (CMAME) (2019, accepted for publication). E-print. arXiv: 1807.08851

10. Hesthaven, J.S., Rozza, G., Stamm, B.: Certified Reduced Basis Methods for Parametrized Partial Differential Equations. SpringerBriefs in Mathematics. Springer, Berlin (2016)

11. Holmes, P., Lumley, J., Berkooz, G.: Turbulence, Coherent Structures, Dynamical Systems and Symmetry. Cambridge University Press, Cambridge (1996)

12. Karniadakis, G., Sherwin, S.: Spectral/hp Element Methods for Computational Fluid Dynamics, 2nd edn. Oxford University Press, Oxford (2005)

13. Lassila, T., Manzoni, A., Quarteroni, A., Rozza, G.: Model order reduction in fluid dynamics: Challenges and perspectives. In: Quarteroni, A., Rozza, G. (eds.) Reduced Order Methods for Modelling and Computational Reduction. MS\&A Modeling, Simulation and Applications, vol. 9, pp. 235-273. Springer International Publishing, Cham (2014)

14. Lovgren, A.E., Maday, Y, Ronquist, E.M.: A reduced basis element method for the steady Stokes problem. ESAIM: Math. Model. Numer. Anal. 40(3), 529-552 (2006)

15. Maday, Y., Ronquist, E.M.: A reduced-basis element method. Comptes Rendus Math. 335(2), 195-200 (2002)

16. Patera, A.T.: A spectral element method for fluid dynamics; laminar flow in a channel expansion. J. Comput. Phys. 54(3), 468-488 (1984)

17. Pitton, G., Rozza, G.: On the application of reduced basis methods to bifurcation problems in incompressible fluid dynamics. J. Sci. Comput. 73, 157-177 (2017)

18. Pitton, G., Quaini, A., Rozza, G.: Computational reduction strategies for the detection of steady bifurcations in incompressible fluid-dynamics: applications to Coanda effect in cardiology. J. Comput. Phys. 344, 534-557 (2017)

19. Quarteroni, A., Rozza, G.: Numerical solution of parametrized Navier-Stokes equations by reduced basis methods. Num. Meth. Part. Diff. Eq. 23(4), 923-948 (2007)

20. Quarteroni, A., Valli, A.: Numerical Approximation of Partial Differential Equations. Springer, Berlin (1994)

21. Rozza, G.: Real-time reduced basis solutions for Navier-Stokes equations: optimization of parametrized bypass configurations. In: ECCOMAS CFD 2006 Proceedings on CD, vol. 676, pp. 1-16 (2006)

22. Wille, R., Fernholz, H.: Report on the first European mechanics colloquium, on the Coanda effect. J. Fluid Mech. 23(4), 801-819 (1965) 
Open Access This chapter is licensed under the terms of the Creative Commons Attribution 4.0 International License (http://creativecommons.org/licenses/by/4.0/), which permits use, sharing, adaptation, distribution and reproduction in any medium or format, as long as you give appropriate credit to the original author(s) and the source, provide a link to the Creative Commons licence and indicate if changes were made.

The images or other third party material in this chapter are included in the chapter's Creative Commons licence, unless indicated otherwise in a credit line to the material. If material is not included in the chapter's Creative Commons licence and your intended use is not permitted by statutory regulation or exceeds the permitted use, you will need to obtain permission directly from the copyright holder. 